Classification

Physics Abstracts

$46.30 \mathrm{~N}-62.20 \mathrm{M}-81.70$

\title{
Caractérisation de l'endommagement de composites tissés à matrice SiC
}

\author{
E. Inghels et J. Lamon (*) \\ Ecole Nationale Supérieure des Mines de Paris, Centre des Matériaux, B.P. 87, 91003 Evry Cedex, France
}

(Reçu le 25 mai 1987, accepté le 10 décembre 1987)

\begin{abstract}
Résumé. - Le comportement mécanique à température ambiante de deux composites bidirectionnels tissés, $\mathrm{C} / \mathrm{SiC}$ et $\mathrm{SiC} / \mathrm{SiC}$ de la $\mathrm{SEP}$, a été étudié à l'aide de plusieurs géométries d'éprouvettes, entaillées ou non. La dégradation a été caractérisée en terme de déformation résiduelle et d'énergie absorbée dans l'ensemble de l'éprouvette. Les résultats des essais de traction et de flexion révèlent des déformations comparables, et une absorption de type volumique, qui est liée à la taille de la zone sollicitée dans les échantillons. Dans les éprouvettes entaillées, la distorsion du réseau de fibres semble être le mécanisme dominant. Les valeurs obtenues pour les paramètres sont comparativement supérieures à celles mesurées en traction et flexion. Dans l'ensemble des essais, les déformations mesurées sont environ 5 fois plus faibles dans le $\mathrm{SiC} / \mathrm{SiC}$ que dans le $\mathrm{C} / \mathrm{SiC}$, mais les énergies absorbées sont comparables dans les deux matériaux.
\end{abstract}

Abstract. - The mechanical behaviour of two 2D woven ceramic composites (namely the $\mathrm{C} / \mathrm{SiC}$ and $\mathrm{SiC} / \mathrm{SiC}$ composites produced by the SEP company), was investigated at room temperature. The analysis of damage was based on residual strain and work of fracture. Similar strains were measured on tensile and flexure specimens. The work of fracture was dependent on the size of the stressed zone. Distorsions of the fibrous structure appeared as the most important cause of damage in the notched specimens and results depended on the specimen geometry. Although residual strains in $\mathrm{C} / \mathrm{SiC}$ are 5 times higher than in $\mathrm{SiC} / \mathrm{SiC}$ composites, comparable work of fracture was obtained with the two materials.

\section{Introduction.}

Les composites céramiques à fibres longues sont des matériaux nouveaux à plus d'un titre, et notamment pour leur comportement mécanique. Leur rupture comprend en général plusieurs phases : la dégradation débute dans la matrice, mais le réseau de fibres préserve l'intégrité du matériau jusqu'à la rupture des fibres. Le comportement mécanique est non linéaire, et il présente un caractère plus ou moins fragile qui dépend de la géométrie des échantillons et des sollicitations [1, 2].

Dans les composites unidirectionnels, des dégradations du réseau de fibres compliquent en général le mode d'endommagement précédent. Ces dégradations peuvent entraîner des ruptures prématurées, notamment par délaminage, ou produisent simplement des distorsions dans le réseau de fibres, quand ce dernier peut supporter une certaine déformation. Ce cas est en particulier celui des deux composites

(*) Adresse actuelle : BATTELLE, Centre de Genève, 7 route de Drize, 1227 Carouge (Genève) Suisse. bidirectionnels à matrice de carbure de silicium, $\mathrm{C} / \mathrm{SiC}$ et $\mathrm{SiC} / \mathrm{SiC}$, qui sont l'objet de cette étude.

Compte-tenu de leur mode de dégradation particulier, dans lequel la structure tissée des matériaux joue notamment un rôle prépondérant, nous avons développé une approche spécifique en vue de caractériser le comportement de ces deux composites. Elle est fondée sur un traitement séparé de la réponse mécanique du matériau aux sollicitations imposées, et de la dégradation accumulée dans l'éprouvette testée. Le premier aspect du comportement a déjà été exposé [3] et il a conduit à définir une loi de comportement local du matériau. Nous nous attachons dans le présent article à caractériser l'endommagement qui apparaît dans le matériau sollicité. Il est exprimé en terme de déplacement résiduel et d'énergie absorbée par les échantillons, qui sont évalués à partir de courbes de comportement déterminées à l'aide de plusieurs types d'éprouvettes. Cette approche a permis de caractériser l'incidence de la déformation irréversible du réseau de fibres sur l'endommagement des deux composites étudiés. 


\section{Etude expérimentale.}

Les deux composites $\mathrm{C} / \mathrm{SiC}$ et $\mathrm{SiC} / \mathrm{SiC}$, produits par la Société Européenne de Propulsion, sont formés d'un réseau de torons de fibres qui est infiltré en phase gazeuse par la matrice de carbure de silicium. Ce réseau, bidirectionnel et orthotrope, est constitué de couches tissées, superposées et identiques entre elles. La figure 1 représente la structure des deux matériaux étudiés. Les modes de tissage sont différents, les torons de SiC étant plus étroitement liés que ceux de carbone, mais dans les deux cas, la porosité importante (10 à $30 \%)$ et répartie de façon non uniforme laisse subsister la structure initiale des torons de fibres. Une estimation des caractéristiques des fibres et de la matrice est donnée dans le tableau I.

L'étude expérimentale du comportement mécanique a utilisé des éprouvettes de traction, de flexion et des éprouvettes entaillées de type SENB, CT et DCB (Fig. 2). Les éprouvettes DCB étaient identiques aux CT, excepté pour la longueur (40 mm). L'orientation des couches par rapport aux sollicitations était telle qu'elles étaient toutes soumises aux
Tableau I. - Caractéristiques des matériaux.

[Properties of the materials.]

\begin{tabular}{|l|c|c|}
\hline \multicolumn{1}{|c|}{ Matériau } & $\mathrm{C} / \mathrm{SiC}$ & $\mathrm{SiC} / \mathrm{SiC}$ \\
\hline Taux volumique de fibres $V_{\mathrm{f}}$ & 0,28 & 0,45 \\
Rayon des fibres $r_{\mathrm{f}}(\mu \mathrm{m})$ & 4 & 6 \\
Module élastique des fibres $E_{\mathrm{f}}(\mathrm{GPa})$ & 180 & 190 \\
Module élastique de la matrice $E_{\mathrm{m}}(\mathrm{GPa})$ & 350 & 350 \\
Cisaillement à l'interface $\tau_{\mathrm{i}}(\mathrm{MPa})$ & 45 & 120 \\
\hline Propriétés des fibres et de la matrice : & & \\
d'après [3] & & \\
Cisaillement à l'interface : & & \\
estimé d'après [4] & & \\
\hline
\end{tabular}

mêmes déformations planes de façon à exclure les ruptures par délaminage. Une des deux orientations de fibres coïncidait toujours avec la direction des sollicitations dominantes. Enfin, le nombre de couches sollicitées était suffisant pour pouvoir considérer que les mesures soient représentatives des carac-
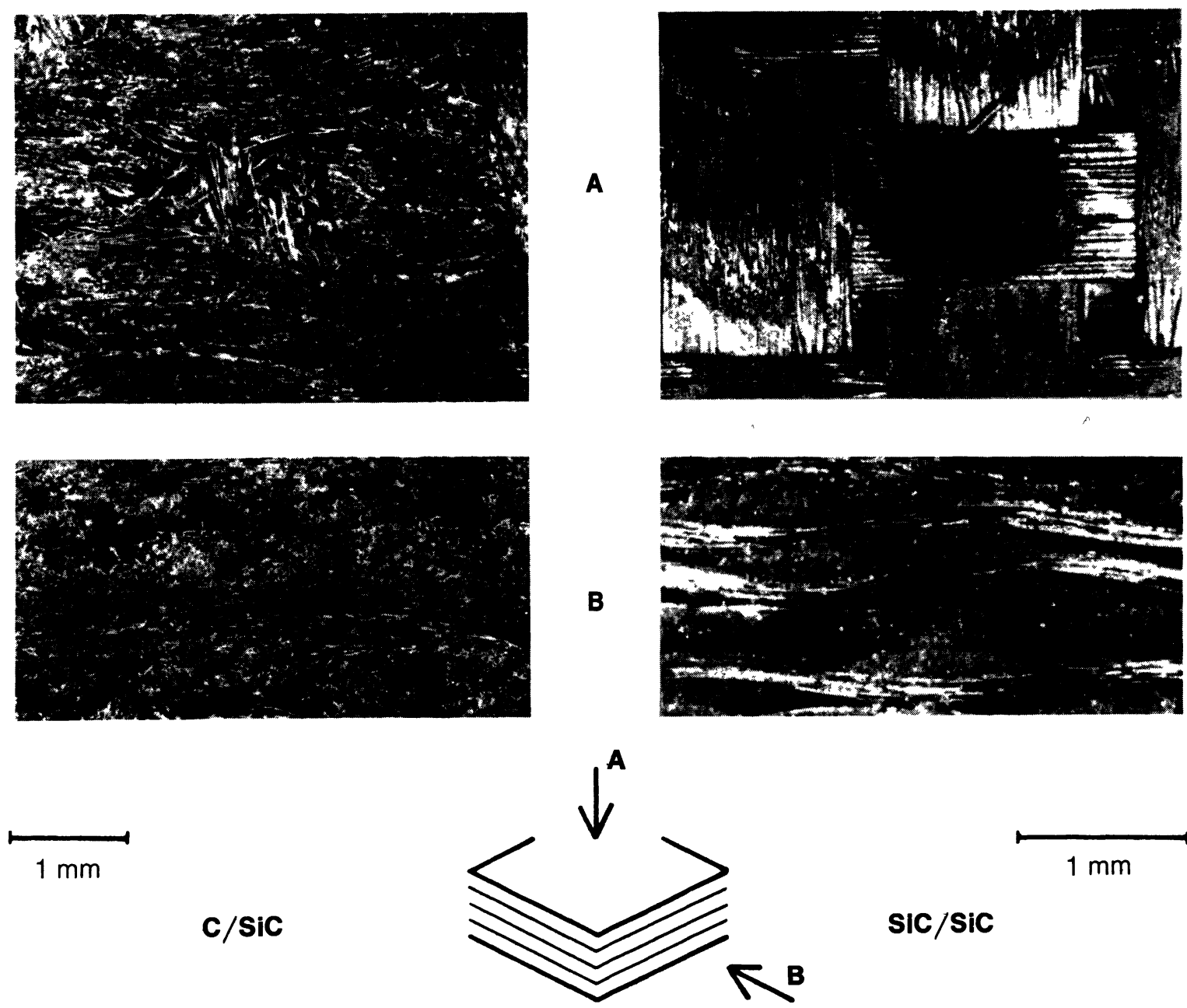

Fig. 1. - Structure des matériaux.

[Structure of the tested materials.] 

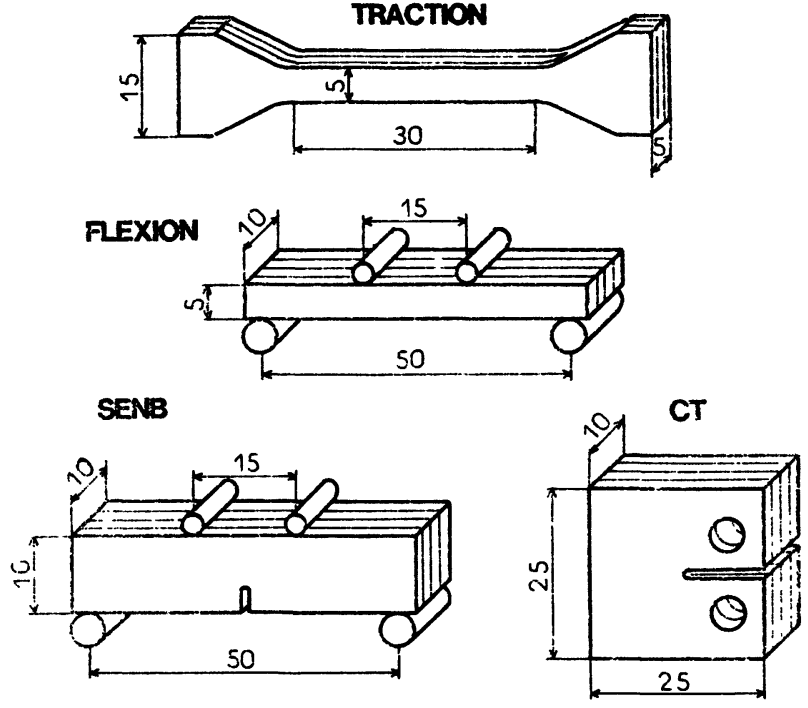

Fig. 2. - Eprouvettes utilisées.

[Tested specimens.]

téristiques moyennes dans le plan d'orthotropie des matériaux (Fig. 2).

Les éprouvettes ont été sollicitées par le déplacement de la traverse d'une machine de traction, à une vitesse de $0,2 \mathrm{~mm} / \mathrm{mn}$. Le comportement mécanique a été représenté par les variations de la charge résultante $P$ en fonction d'un déplacement $\delta$ mesuré entre deux points fixes. Ils sont matérialisés par des couteaux collés, soit sur la face en tension des éprouvettes de traction et de flexion, soit de part et d'autre des lèvres de l'entaille dans les autres types d'éprouvettes. Des déchargements partiels suivis d'un rechargement ont été effectués de façon régulière au cours des essais. Ils permettent d'estimer dans $\delta$ la contribution des déformations résiduelles de l'éprouvette.

\section{Mode d'endommagement.}

Les courbes effort-déplacement obtenues sont représentées sur la figure 3 . Elles comportent trois parties :

- une phase initiale linéaire qui reflète le comportement élastique des composites ;

- puis une partie non linéaire de pente plus faible, qui traduit notamment l'existence d'un endommagement dans la matrice, l'intégrité du matériau étant assurée par les fibres ;

- enfin une décroissance de l'effort au-delà du maximum, qui résulte de la rupture des fibres.

Le comportement est similaire quelle que soit l'éprouvette, excepté dans la phase de rupture finale. Celle-ci est rapide dans les éprouvettes de traction et de flexion (entaillées ou non) et stable dans les éprouvettes CT et DCB. Un déplacement

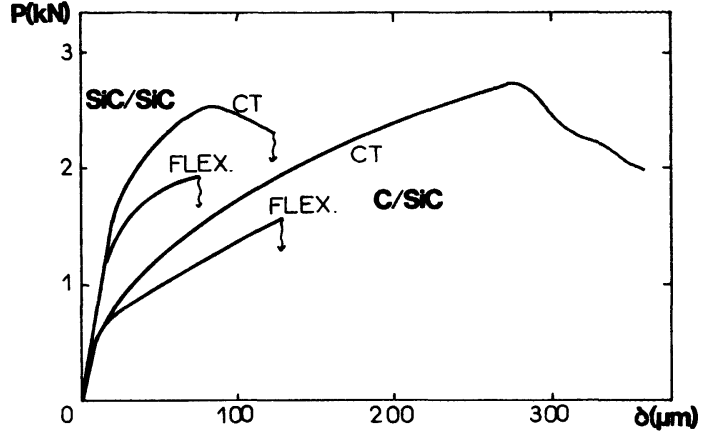

[Load-displacement curves.]

Fig. 3. - Allure des courbes charge-déplacement.

résiduel est détecté au début de la partie non linéaire. Il croît ensuite de façon plus ou moins régulière tout au long de la mise en charge. Etant 2 à 3 fois plus élevé dans le $\mathrm{C} / \mathrm{SiC}$ que dans le $\mathrm{SiC} / \mathrm{SiC}$, il reflète la différence de déformabilité entre les deux matériaux, mise en évidence par les courbes effort-déplacement (Fig. 3).

L'observation des surfaces rompues a montré que les deux matériaux se comportent comme des ensembles d'éléments indépendants, constitués chacun d'un toron de fibres et de la matrice qui y est déposée [3]. Le processus d'endommagement de chacune de ces «baguettes ", où une fissuration multiple de la matrice précède la rupture des fibres, permet en outre de les assimiler à des composites unidirectionnels sollicités en traction $[2,4]$. Les fissures de la matrice, perpendiculaires aux fibres et réparties régulièrement, sont plus nombreuses dans le $\mathrm{C} / \mathrm{SiC}[3]$.

L'ouverture des fissures précédentes, ainsi que la faiblesse de certaines interfaces, provoquent un allongement résiduel des baguettes endommagées. Ces allongements entraînent des distorsions de la structure tissée, que peuvent amplifier des glissements des baguettes les unes par rapport aux autres. Ces mécanismes laissent peu de traces visibles sur les éprouvettes, mais les distorsions qu'ils engendrent déforment de façon irréversible les éprouvettes. Ainsi les barreaux sollicités en flexion présentent une flèche permanente, qui résulte de courbures résiduelles situées au droit des points d'application de la charge. Les éprouvettes entaillées présentent quant à elles une ouverture angulaire de l'entaille, ainsi qu'un déplacement irréversible dans la zone proche du front de rupture, qui tend à séparer les deux surfaces rompues.

\section{Caractérisation de l'endommagement.}

4.1 ESSAIS DE TRACTION ET DE FLEXION. L'endommagement est caractérisé par la déformation résiduelle moyenne $\varepsilon_{\mathrm{r}}$, déduite de la partie irréversible du déplacement $\delta$, et par l'énergie 
absorbée dans l'ensemble de l'éprouvette $W_{\mathrm{f}}$. Cette dernière est donnée par la différence entre le travail fourni par le dispositif et l'énergie élastique de l'éprouvette. En plus des grandeurs mesurées durant l'essai, son évaluation nécessite la connaissance du déplacement du point d'application de la charge $\delta_{\mathrm{p}}$. Celui-ci comporte une partie élastique, qui est évaluée dans le cas d'un milieu isotrope linéaire, et une partie résiduelle déduite de $\delta$ grâce à une schématisation de la déformée résiduelle globale (Figs. 4 et 5). La zone endommagée des éprouvettes

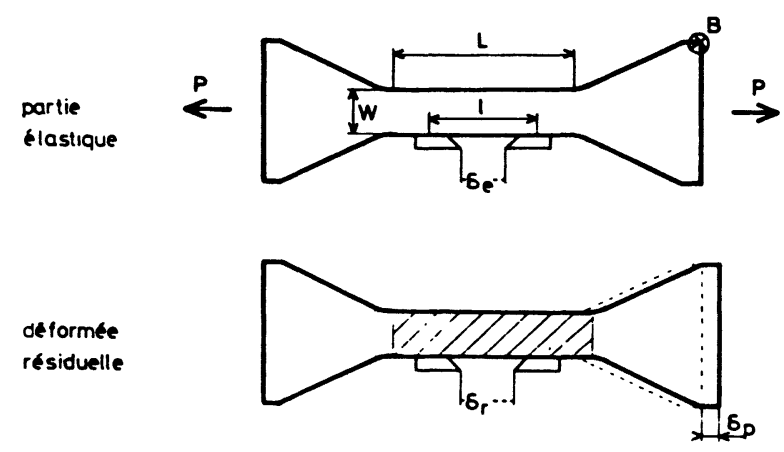

Fig. 4. - Schématisation de la déformée en traction.

[Displacement assumed in tensile test specimen.]

partie elastique

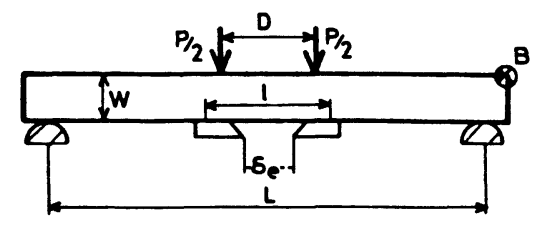

deforme residuelle

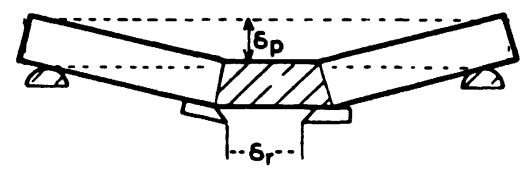

Fig. 5. - Schématisation de la déformée en flexion.

[Displacement assumed in flexure test specimen.]

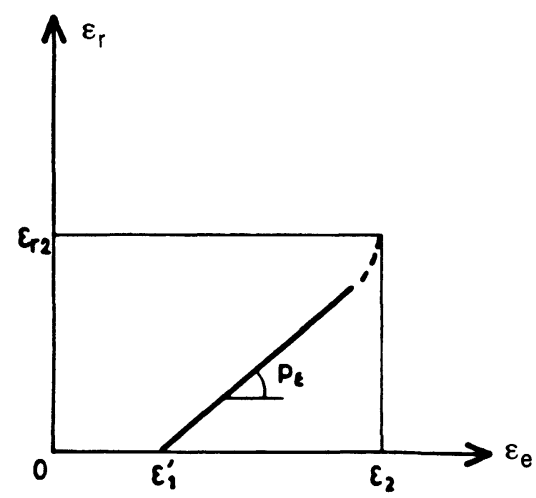

Fig. 6. - Exploitation des grandeurs de dégradation. est supposée limitée au fût en traction, et à la partie située entre les points où la charge est appliquée en flexion. Dans ce dernier cas, la déformation est choisie maximale sur la face en tension, et nulle sur celle en compression. La déformée ainsi schématisée s'est révélée en bon accord avec des mesures directes de profils d'éprouvettes effectuées au cours d'essais de flexion. La déformation résiduelle et l'énergie absorbée ont été évaluées par les expressions suivantes (avec les notations des Figs. 4 et 5) : en traction:

$$
\varepsilon_{\mathrm{r}}=\delta_{\mathrm{r}} / l \quad W_{\mathrm{f}}=\int P \mathrm{~d} \delta-P \delta_{\mathrm{e}}
$$

en flexion :

$$
\begin{aligned}
& \varepsilon_{\mathrm{r}}=\delta_{\mathrm{r}} / l \quad W_{\mathrm{f}}=\frac{(L-D)}{12 W l}(L+2 D) \times \\
& \times\left[\int_{0}^{\delta} P \mathrm{~d}\left(\delta_{\mathrm{e}}+\frac{3 l}{2(L+2 D)} \delta_{\mathrm{r}}\right)-P \delta_{\mathrm{e}}\right] .
\end{aligned}
$$

La mise en charge des éprouvettes est décrite par la déformation élastique $\varepsilon_{\mathrm{e}}$, qui représente la partie élastique du déplacement $\delta$. Les grandeurs $\varepsilon_{\mathrm{r}}$ et $W_{\mathrm{f}}$ évoluent en fonction de $\varepsilon_{\mathrm{e}}$ de manière comparable : elles débutent à partir d'une déformation élastique $\varepsilon_{1}^{\prime}$, puis augmentent de façon sensiblement linéaire. Cette croissance s'accélère en général peu avant la rupture brutale. Elles sont ici caractérisées par le seuil $\varepsilon_{1}^{\prime}$, et par le taux d'accroissement moyen et la valeur à rupture de chacune des grandeurs (Fig. 6). Les résultats expérimentaux sont réunis dans les tableaux II et III.

Le début de l'endommagement, caractérisé ici par $\varepsilon_{1}^{\prime}$, a précédemment été mesuré par la limite $\varepsilon_{1}$ du domaine linéaire de la courbe de comportement contrainte-déformation élastique [3]. Il existe une corrélation entre les deux paramètres (Fig. 7), et la déformation $\varepsilon_{1}^{\prime}$ représente environ $60 \%$ de $\varepsilon_{1}$ dans le cas du C/SiC et $80 \%$ dans le cas du $\mathrm{SiC} / \mathrm{SiC}$. Cet écart systématique entre deux paramè-

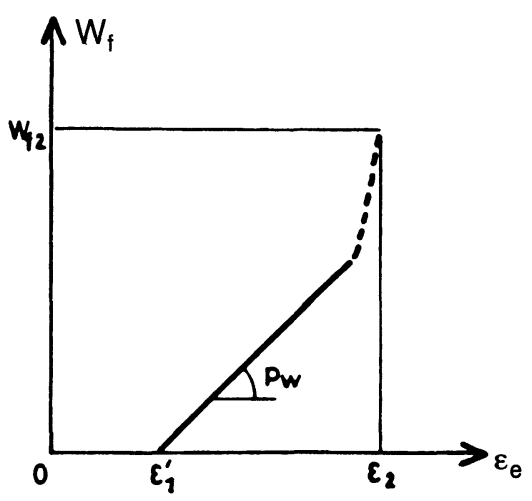

[Evolution of characteristics of damage.] 
Tableau II. - Valeurs expérimentales des paramètres caractérisant la déformation résiduelle (exprimées par valeur moyenne, (écart-type)).

[Experimental results obtained for residual strain, expressed as average values (standard deviation).]

\begin{tabular}{|l|c|c|c|}
\cline { 2 - 4 } \multicolumn{1}{c|}{} & paramètre & $p_{\varepsilon}$ & $\varepsilon_{\mathrm{r} 2}\left(10^{-3}\right)$ \\
\hline $\mathrm{C} / \mathrm{SiC}$ & traction & $3,2(0,8)$ & $3,6(1,2)$ \\
& flexion & $3,0(0,8)$ & $7,6(2,1)$ \\
\hline $\mathrm{SiC} / \mathrm{SiC}$ & $\begin{array}{c}\text { traction }+ \\
\text { flexion }\end{array}$ & $0,8(0,6)$ & $1,3(1,2)$ \\
\hline
\end{tabular}

Tableau III. - Valeurs expérimentales des paramètres caractérisant l'énergie absorbée.

[Experimental results for the works of fracture, expressed as average value (standard deviation).]

\begin{tabular}{|l|c|c|c|}
\cline { 2 - 4 } \multicolumn{1}{c|}{} & paramètre & $\begin{array}{c}P_{\mathrm{w}} / \mathrm{vol} \\
\left(\mathrm{MJ} / \mathrm{m}^{3}\right)\end{array}$ & $\begin{array}{c}W_{\mathrm{f}} / \mathrm{sect} \\
(\mathrm{KJ} / \mathrm{m})\end{array}$ \\
\hline $\mathrm{C} / \mathrm{SiC}$ & traction & $141(51)$ & $5,2(2,8)$ \\
& flexion & $22(6)$ & $2,9(1,1)$ \\
\hline \multirow{2}{*}{$\mathrm{SiC} / \mathrm{SiC}$} & traction & $101(65)$ & $1,8(1,5)$ \\
& flexion & $10(1)$ & $2,3(2,0)$ \\
\hline
\end{tabular}

tres qui caractérisent un même phénomène peut être attribué à une plus grande sensibilité des grandeurs $\varepsilon_{\mathrm{r}}$ et $W_{\mathrm{f}}$ pour détecter la présence d'un endommagement.

Compte-tenu de la dispersion des résultats, les évolutions de la déformation résiduelle mesurées en traction et flexion peuvent être considérées comme comparables. Les déformations finales sont en outre peu différentes de la valeur obtenue en extrapolant la phase linéaire jusqu'à la rupture. La phase de dégradation rapide, attribuable aux premières ruptures de fibres, joue donc un rôle limité dans les déformations mesurées.

La contribution de l'allongement irréversible des baguettes est estimée à partir des faciès de rupture, où elle est représentée par l'ouverture des fissures présentes dans la matrice. L'ouverture de chaque fissure est de l'ordre de $1 \mu \mathrm{m}$ dans les deux matériaux. Une dizaine de fissures sont en général observées autour des surfaces de rupture. L'allongement des baguettes peut alors expliquer seul les valeurs de la déformation $\varepsilon_{\mathrm{r} 2}$ dans le $\mathrm{SiC} / \mathrm{SiC}$ qui est mesurée sur une distance d'environ $1 \mathrm{~cm}$. Par contre dans le $\mathrm{C} / \mathrm{SiC}$ où la déformation est cinq fois plus importante, il faut admettre l'existence de glissements entre les baguettes. Cette différence de comportement entre les deux tissus est conforme à leurs structures respectives, les baguettes du $\mathrm{SiC} / \mathrm{SiC}$ étant mieux liées entre elles (Fig. 1). L'ouverture de chaque fissure provient en partie d'une relaxation des efforts dans la matrice rompue, dont la contribution peut être évaluée théoriquement à partir des propriétés des constituants [4]. Les valeurs ainsi obtenues sont de $0,1 \mu \mathrm{m}$; dans les deux matériaux. L'essentiel des ouvertures mesurées doit donc être attribué à des glissements aux interfaces
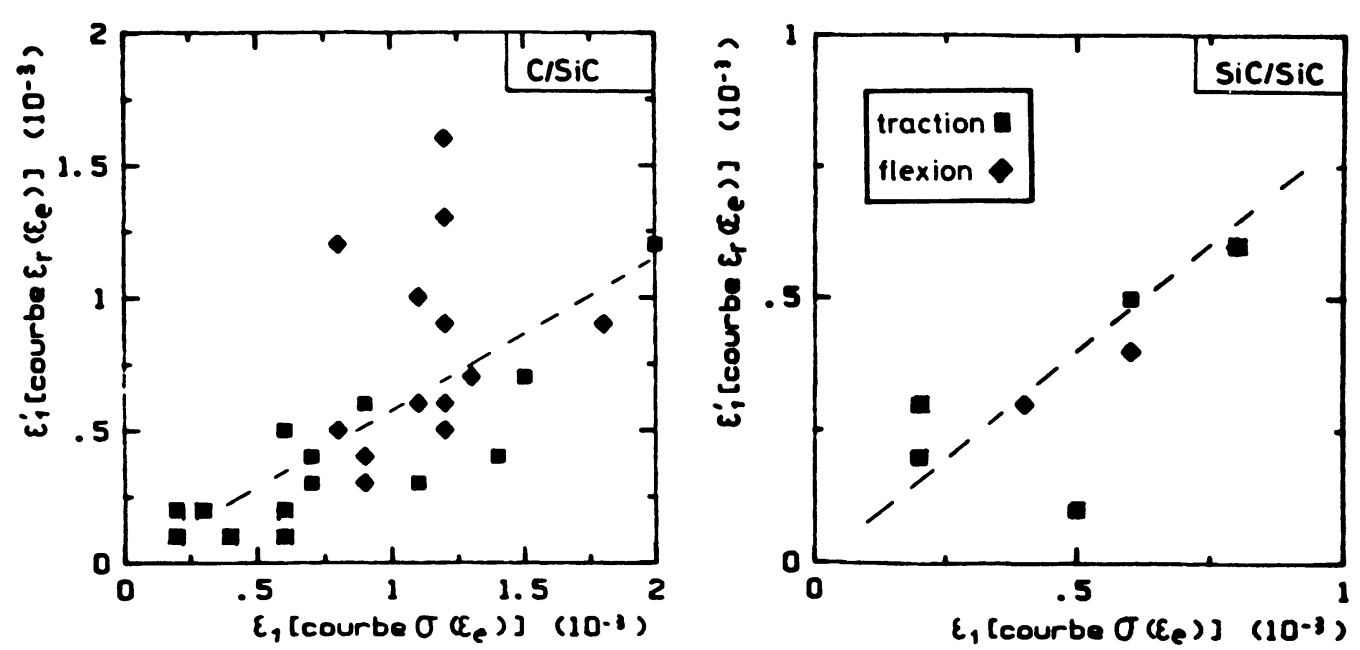

Fig. 7. - Comparaison du début de non-linéarité et du début d'endommagement dans le $\mathrm{C} / \mathrm{SiC}$ et le $\mathrm{SiC} / \mathrm{SiC}$ (éprouvettes de traction et de flexion).

[Damage initiation versus linearity limit of the $\sigma\left(\varepsilon_{\mathrm{c}}\right)$ curve (tensile and flexure tests on $\mathrm{C} / \mathrm{SiC}$ and $\mathrm{SiC} / \mathrm{SiC}$ specimens).] REVUE DE PHYSIQUE APPLIQUÉE. - T. 23, N`3, MARS 1988 
qui provoquent des déplacements des fibres les unes par rapport aux autres.

L'énergie absorbée est due à la création de surfaces au sein de la matrice, et à une dissipation par frottement liée aux déplacements irréversibles se produisant dans le matériau. Ces mécanismes sont similaires dans les essais de traction et de flexion. Les valeurs du taux d'accroissement $p_{\mathrm{w}}$ plus faibles en flexion sont alors attribuées à la différence de taille des zones endommagées dans les deux types d'éprouvettes. L'écart qui résulte de cet effet de la géométrie a été évalué en considérant que la variation $W_{\mathrm{f}}\left(\varepsilon_{\mathrm{e}}\right)$ mesurée en traction représente une loi d'absorption volumique en fonction de la sollicitation locale. Le principe de la méthode est décrit sur la figure 8 , l'énergie absorbée en flexion étant obtenue
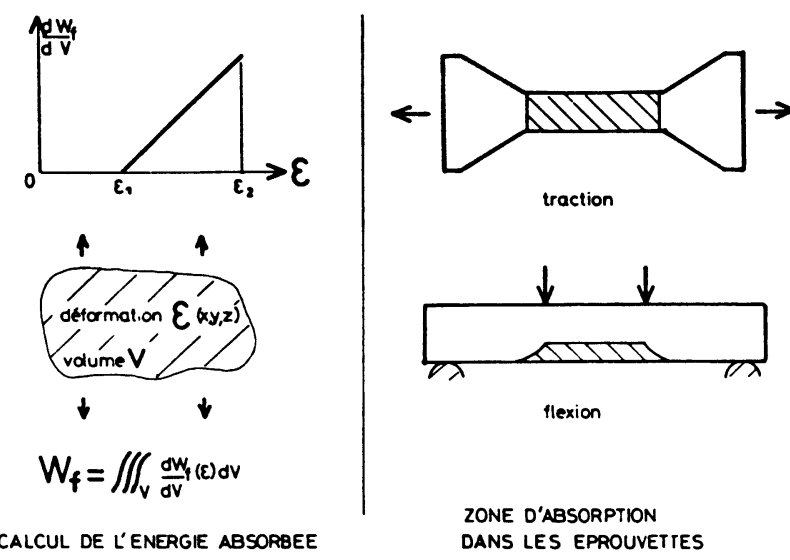

Fig. 8. - Evaluation de l'énergie absorbée pour deux géométries d'éprouvettes.

[Determination of work of fracture for the two specimen geometries.]

par intégration de la loi locale précédente sur la répartition de déformation existant dans un milieu isotrope élastique. Le rapport des taux d'accroissement $p_{\mathrm{w}}$ mesurés respectivement en flexion et en traction s'exprime ainsi de façon approchée, et avec les notations des figures 5 et 8 par:

$$
\frac{D}{2 L}\left[1-\left(\frac{\varepsilon_{1}^{\prime}}{\varepsilon_{2}}\right)^{2}\right] \text {. }
$$

Il est ici compris entre 0,1 et 0,15 dans les deux matériaux, ce qui est conforme aux résultats expérimentaux du tableau III. A cette absorption de type volumique au cours de l'endommagement, s'ajoute un terme de surface au moment de la rupture, qui représente la différence entre la valeur $W_{\mathrm{f} 2}$ et l'énergie à rupture extrapolée à partir du taux d'accroissement $p_{\mathrm{w}}$. La dispersion des mesures ne permet pas de préciser la valeur de ce terme. Il doit donc être considéré comme inférieur à $1 \mathrm{KJ} / \mathrm{m}^{2}$.
4.2 ESSAIS SUR ÉPROUVETTES ENTAILlÉES. L'endommagement est caractérisé par le déplacement irréversible à l'avant de la rupture $\delta_{\mathrm{f}}$ et par l'énergie absorbée $W_{\mathrm{f}}$ qui est définie de la même façon que pour les essais de traction et flexion. Elles ont été évaluées à partir de la charge $P$ et du déplacement aux lèvres de l'entaille $\delta$, moyennant certaines hypothèses sur la déformée globale des éprouvettes. Ces dernières s'appuient sur des relations établies pour des milieux isotropes élastiques [5-7], et sur une déformée résiduelle qui est schématisée sur la figure 9 . Comme le proposent certains auteurs $[8,9]$, le déplacement $\delta_{\mathrm{f}}$ et l'ouverture angulaire de l'entaille sont pris en compte en introduisant un centre de rotation fictif dans le ligament.

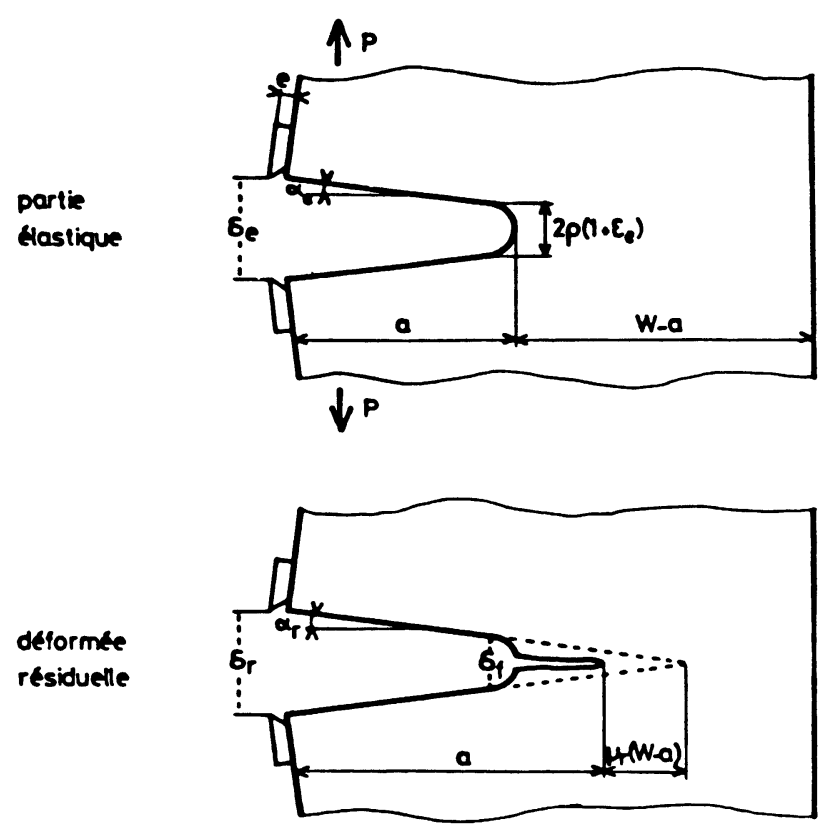

Fig. 9. - Schématisation de la déformée des éprouvettes entaillées.

[Displacements assumed in notched specimens.]

Sa position est repérée par un paramètre $\mu_{\mathrm{r}}$, pris ici égal à 0,4 . Cette valeur constitue une moyenne de celles utilisées pour divers matériaux [8,9]. En outre, elle prévoit des angles d'ouverture de l'entaille qui sont comparables à ceux mesurés directement sur des éprouvettes CT et DCB en cours d'essais (Fig. 10).

La propagation de la rupture débute peu avant le point de charge maximale. Elle est brutale dans les éprouvettes SENB et en partie stable dans les éprouvettes CT et DCB. Les grandeurs $\delta_{f}$ et $W_{\mathrm{f}}$ sont analysées différemment de part et d'autre du maximum de charge. Dans la phase précédant la propagation, seules les valeurs de $\delta_{\mathrm{f}}$ et $W_{\mathrm{f}}$ mesurées au niveau de la charge maximale sont examinées (Tab. IV). Les résultats présentent des disparités en fonction de la géométrie utilisée, qui affectent 

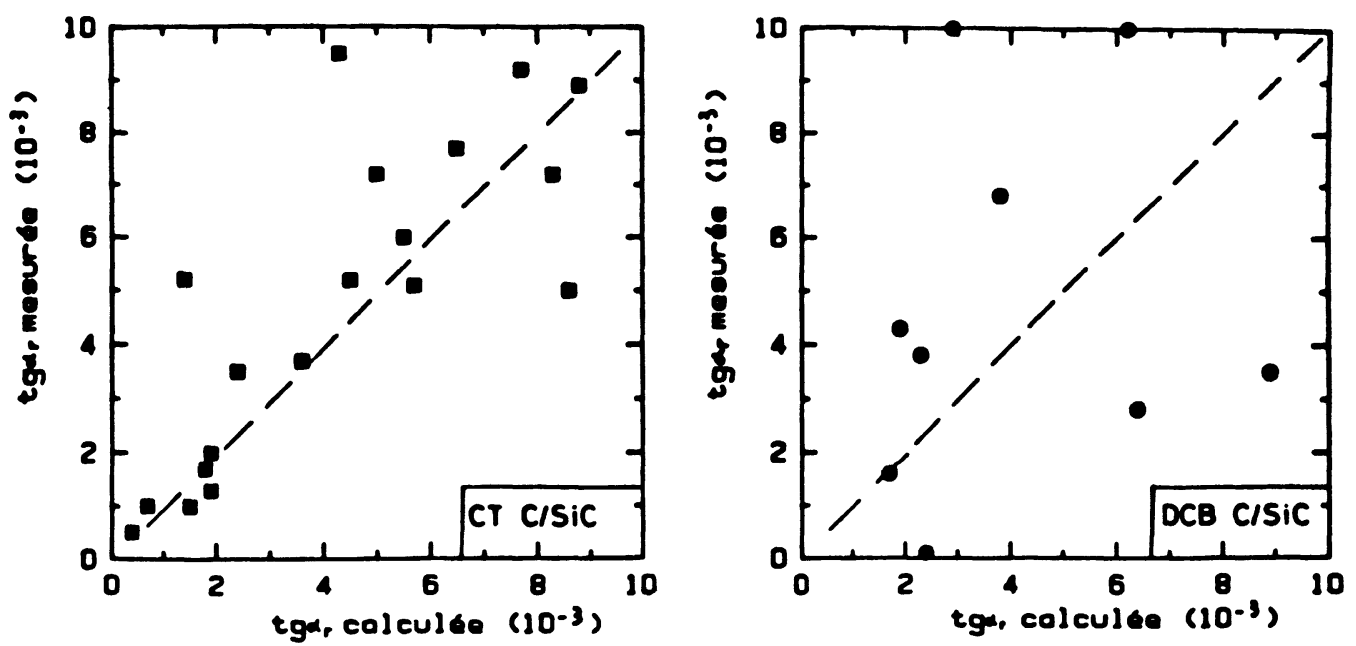

Fig. 10. - Etalonnages de l'angle d'ouverture résiduelle dans le $\mathrm{C} / \mathrm{SiC}$.

[Measured and calculated values for the residual crack opening angle in $\mathrm{C} / \mathrm{SiC}$ composites.]

Tableau IV. - Valeurs expérimentales du COD et de l'énergie absorbée dans les éprouvettes entaillées, mesurées au maximum de charge (exprimées par valeur moyenne (écart-type)).

[Experimental results for COD and work of fracture obtained in notched specimens, at the point of maximum load, expressed as average value (standard deviation).]

\begin{tabular}{|l|c|r|r|}
\cline { 2 - 4 } \multicolumn{1}{c|}{} & paramètre & $\delta_{\mathrm{f}}(\mu \mathrm{m})$ & \multicolumn{1}{c|}{$W_{\mathrm{f}}(\mathrm{J} / \mathrm{m})$} \\
\hline \multirow{4}{*}{$\mathrm{C} / \mathrm{SiC}$} & SENB & $20(2)$ & $5,8(2,2)$ \\
& $\mathrm{CT}(W=40)$ & $61(14)$ & $33,8(8,5)$ \\
& DCB & $78(20)$ & $26,5(8,1)$ \\
\hline \multirow{3}{*}{$\mathrm{SiC} / \mathrm{SiC}$} & SENB & $11(6)$ & $5,2(3,1)$ \\
& CT $(W=20)$ & $7(4)$ & $4,0(3,1)$ \\
& DCB & $34(15)$ & $19,1(10,0)$ \\
\hline
\end{tabular}

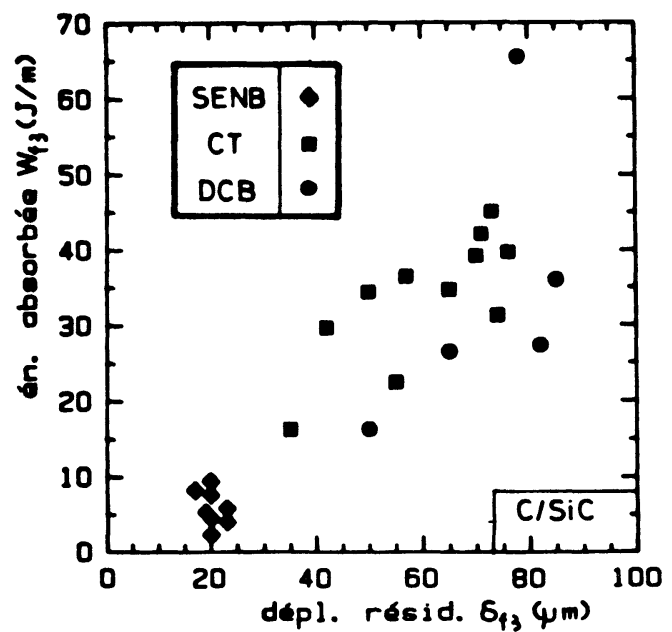

cependant $\delta_{\mathrm{f}}$ et $W_{\mathrm{f}}$ de façons comparables. Une corrélation apparaît ainsi entre les deux grandeurs (Fig. 11), qui illustre le fait que $\delta_{\mathrm{f}}$ et $W_{\mathrm{f}}$ représentent un même ensemble de dégradations.

$\mathrm{Au}$ cours de la propagation, les évolutions de $\delta_{\mathrm{f}}$ et $W_{\mathrm{f}}$ sont décrites en fonction de l'avancée de rupture $a$, qui est évaluée à partir de la complaisance par une méthode décrite précédemment [3]. Le déplacement $\delta_{\mathrm{f}}$ demeure sensiblement constant, mais il est très différent dans les deux matériaux (70 $\mu \mathrm{m}$ dans le $\mathrm{C} / \mathrm{SiC}$ et $10 \mu \mathrm{m}$ dans le $\mathrm{SiC} / \mathrm{SiC}$ ). L'énergie absorbée augmente quant à elle de façon sensiblement linéaire, avec des taux d'accroissement voisins dans les deux composites, d'environ $4,5 \mathrm{KJ} / \mathrm{m}^{2}$ dans le $\mathrm{C} / \mathrm{SiC}$ et de 5 à $6 \mathrm{KJ} / \mathrm{m}^{2}$ dans le $\mathrm{SiC} / \mathrm{SiC}$.

La contribution des baguettes à l'énergie de rupture provient essentiellement de la dégradation de la matrice dans une zone confinée autour de la

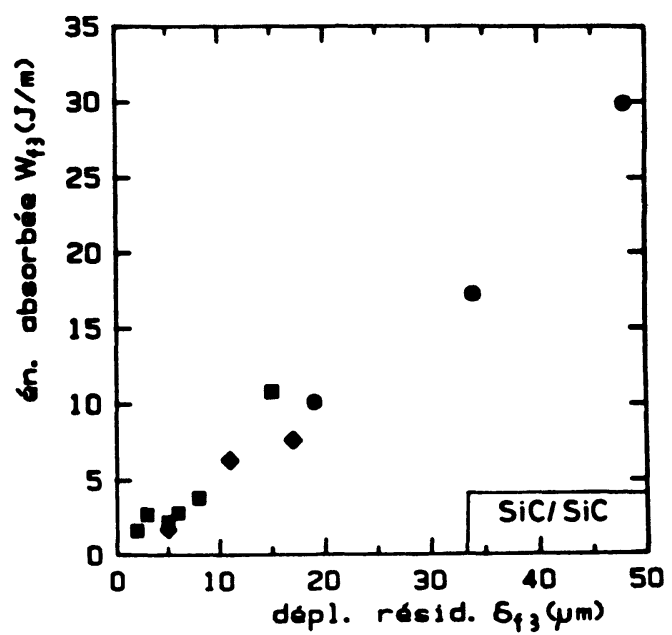

Fig. 11. - Comparaison des ouvertures en fond d'entaille et des énergies absorbées (mesurées pour la charge maximale).

[Crack opening displacement versus work of fracture in notched specimens, measured at maximum load.] 
surface de rupture et du frottement des fibres rompues qui sont extraites de la matrice. D'après les résultats obtenus en traction et flexion, le premier terme ne dépasse guère $10 \mathrm{~J} / \mathrm{m}$ à l'amorçage de la rupture. Le travail d'extraction de fibres peut être évalué à partir des propriétés des constituants et de l'interface [10]. Les données du tableau I fournissent des dissipations d'environ $200 \mathrm{~J} / \mathrm{m}^{2}$ dans les deux matériaux. Ces prévisions mettent en évidence le fait que l'essentiel de l'énergie est absorbée dans les éprouvettes entaillées par la déformation globale de la structure. Un tel résultat est en outre cohérent avec les écarts observés tant entre les différentes géométries qu'entre les deux matériaux. Ainsi, les valeurs mesurées au début de la propagation augmentent avec le volume total de l'éprouvette (les CT sont plus grandes que les SENB mais plus petites que les DCB), comme a priori l'ampleur des déplacements irréversibles au sein du matériau. Par ailleurs, la meilleure résistance du tissu de $\mathrm{SiC} / \mathrm{SiC}$ vis-à-vis des distorsions peut expliquer que le travail à fournir pour le déformer soit du même ordre que dans le $\mathrm{S} / \mathrm{SiC}$, bien que les déformations soient plus importantes dans ce dernier.

\section{Conclusion.}

La dégradation des matériaux $\mathrm{C} / \mathrm{SiC}$ et $\mathrm{SiC} / \mathrm{SiC}$ a été caractérisée en terme de déplacements résiduels et d'énergie absorbée, qui illustrent respectivement les modifications géométriques du milieu et la résistance qu'il y oppose. Ces grandeurs recouvrent à la fois l'endommagement à l'intérieur des baguettes et les dégradations de la structure tissée elle-même. Le premier mécanisme s'avère reproductible d'une géométrie à l'autre, et sa contribution dépend uniquement de la répartition volumique des sollicitations. Par contre, le terme lié à la structure varie fortement avec la géométrie, et d'une façon qui reste difficile à quantifier. Il peut être en outre très supérieur au terme précédent. Les diverses géométries font néanmoins apparaître que le $\mathrm{SiC} / \mathrm{SiC}$ se déforme beaucoup moins que le $\mathrm{C} / \mathrm{SiC}$, mais qu'en raison de sa structure plus résistante, le travail de déformation à lui fournir est du même ordre que dans le $\mathrm{C} / \mathrm{SiC}$.

\section{Remerciements.}

Ce travail a été effectué dans le cadre des contrats DRET n 82.305 et 84.082 .

\section{Bibliographie}

[1] Fitzer, E., Schlichting, J., Fiber reinforced refractory oxides, High Temp. Sci. 13 (1980) 149-172.

[2] Marshall, D. B., Evans, A. G., Failure mechanisms in ceramic fiber/ceramic matrix composites, J. Am. Ceram. Soc. 68 (1985) 225-231.

[3] INGHels, E., LAmon, J., Comportement mécanique de composites tissés à matrice SiC. Comptesrendus JNC5 (Editions Pluralis) septembre 1986, pp. 329-342.

[4] Aveston, J., Cooper, G. A., Kelly, A., Single and multiple fracture, in Properties of fiber composites (IPC science and technology press, Guildford, Surrey) 1971, pp. 15-24.

[5] « Standard test method for plane-strain fracture toughness of metallic materials ", American Standard Testing Methods E 399-81 (1981) 588-618.
[6] SHANG-Xian, W., Crack length calculation formula for three point bend specimens, Intern. J. Fract. 24 (1984) R 33-R 35.

[7] ROBERTS, E., Elastic crack-edge displacements for the compact tension specimen, Mater. Res. Stand. 2 (1969) 27.

[8] Matsoukas, G., Cotterell, B., Mai, Y. W., On the plastic rotation constant used in standard COD tests, Intern. J. Fract. 26 (1984) R 49-R 53.

[9] Chong, K. P., Kuruppu, M. D., New specimen for fracture toughness determination for rock and others materials, Intern. J. Fract. 26 (1984) R 59R 62.

[10] Wells, J. K., Beaumont, P. W. R., Debonding and pull-out processes in fibrous composites, $J$. Mater. Sci. 20 (1985) 1275-1284. 